\title{
fMRI BOLD Signal Changes in Elite Swimmers While Viewing Videos of Personal Failure
}

\author{
Henry Davis IV • Mario Liotti • Elton T. Ngan • \\ Todd S. Woodward • Jared X. Van Snellenberg • \\ Sari M. van Anders • Aynsley Smith • Helen S. Mayberg
}

Received: 10 August 2007 / Accepted: 10 December 2007 / Published online: 17 January 2008

(C) Springer Science + Business Media, LLC 2007

\begin{abstract}
Athletes who fail are susceptible to negative affect (NA) and impaired future performance. Functional magnetic resonance imaging (fMRI) studies have identified prefrontal, anterior cingulate, and limbic activations following negative mood provocation. Little is known about the neural correlates of negative self-reference (SR), especially in athletes. Even less is known about the neural correlates of the effects of
\end{abstract}

\section{H. Davis IV $(\bowtie)$}

Swimming Canada,

Suite 354, 401- 9th Avenue S.W.,

Calgary, Alberta T2P 3C5, Canada

e-mail: hapdavis@gmail.com

\section{Liotti}

Department of Psychology, Simon Fraser University,

Burnaby, British Columbia, Canada

E. T. Ngan • T. S. Woodward

Schizophrenia Cognition and Imaging Lab,

Department of Psychiatry, University of British Columbia,

2C1-2255 Wesbrook Mall,

Vancouver, British Columbia V6T 2A1, Canada

J. X. Van Snellenberg

Department of Psychology, Columbia University,

1190 Amsterdam Ave.,

New York, NY 10027, USA

S. M. van Anders

Department of Psychological and Brain Sciences, Indiana University,

1101 East Tenth Street,

Bloomington, IN 47405, USA

A. Smith

Sport Medicine Clinic, Mayo Clinic,

200 First Street Southwest,

Rochester, MN 55905, USA

H. S. Mayberg

Emory University School of Medicine,

Atlanta, GA 30322, USA cognitive intervention (CI) in modifying negative SR and NA in this population. In an fMRI study, 13 athletes watched a video of their own career-threatening defeat in two controlled blocks. Between fMRI blocks, they received a 20-min CI designed to assist in event reappraisal and planning for future performance. Relative increases post-CI were seen in premotor (BA6) and sensorimotor (BA4/1) cortices. Correlated with mood ratings, relatively higher pre-CI levels were seen in the ventromedial prefrontal cortex, the right dorsomedial prefrontal cortex (PFC; BA10), the right dorsolateral PFC (BA45), the anterior cingulate, and the right parahippocampus. CI may counteract the detrimental effects of NA and negative SR on premotor and motor activity.

Keywords Self-reference $\cdot$ Emotion .

Cognitive neuroscience $\cdot$ Cognitive intervention

Elite athletes commonly experience sadness and subjective distress after failing in major competition. In turn, subjective distress may yield poorer sport performance (Beedie et al. 2000; LeUnes and Burger 2000). Negative affect (NA) is a term (without causal connotation) for transient subjective distress and sadness.

Dramatic failures are common both in selection for and in competing at major international events; in most cases failure is nothing more than motor execution error: Skills that have been previously over-learned are not executed adequately to produce success. Amateur athletes compete for selection to their national team roughly once a year. The majority, although ranked in the top five in their country and top 40 in the world, are never selected to an Olympic team; not achieving selection regularly results in sadness. Failure-based NA is recurrently experienced by more than half of the world's best athletes. 
Failure to achieve as a national team athlete may trigger NA and a breakdown in emotional self-regulation. Indeed, the leading cause of retirement from amateur sport is failure to qualify for a team. Almost $25 \%$ of athletes report depressive symptoms following failure-based retirement (Baillie and Danish 1992).

Most national team athletes are younger than age 25 . Overall, sport-active young people may have an incidence of sadness and depression that is no greater than that found in the general population (Sanders et al. 2000). However, for Olympic-level athletes there may be a different story. The US Olympic Training Center indicated that possibly $85 \%$ of elite athletes experience serious psychological problems, including those related to NA (Carrm et al. 2005).

Although experience suggests that these negative states are over-represented among elite athletes, their prevalence and implications have not been documented. Happy athletes generally do well, while unregulated NA-NA that is not controlled by appropriate coping responses-has multiple negative repercussions for performance (Fazackerley et al. 2004; Lane et al. 2001; Totterdell and Leach 2001). Several important questions remain unanswered. Would NA after one failed competition predict a similarly inadequate performance in the next competitive event, especially when an athlete retains negative personal memories? What would be the neural and biomechanical mechanisms for repetitive failure?

\section{Neuroimaging of limbic-cortical interactions in mood regulation}

A fuller understanding of neural systems underlying NA would advance empirically-supported intervention. Recent findings in functional brain imaging have enhanced our knowledge of mood circuits that are invoked with the expression and regulation of NA. Earlier studies that used sadness induction and subject-specific autobiographical memory (Mayberg et al. 1999) had illustrated the reciprocal nature of limbic-cortical interactions with NA in healthy subjects and depressives. The central conclusion was that reciprocal changes in subgenual anterior cingulate cortex (ACC) and dorsolateral prefrontal cortex (PFC) play a major role in the expression and regulation of sadness in health and disease.

Reviewing performance is a form of autobiographic review. ACC activation has been seen during NA induction when subjects view sadness in others (Gotlib et al. 2005). Similar effects are predicted from reviewing personal events.

NA suppression, reappraisal, and regulation studies have generally documented an interplay between limbic and cortical systems (Beauregard et al. 2001; Ochsner et al. 2002; Phan et al. 2005). Emotion regulation studies typically achieve mood change by showing emotionally arousing pictures and videos, not by showing self-referent stimuli.
Based on several authors' research (Eugene et al. 2003; Northoff et al. 2006; Ochsner et al. 2002, 2004, 2005), one would expect the regulation of self-relevant NA to be associated with activations of the medial prefrontal cortex (mPFC), ACC, and hippocampus. Evidence suggests differential responding of the amygdala (for regulation of emotion) and frontal areas (for regulation of cognition) depending on whether the subject is asked to increase a positive mood or a negative one (Ochsner et al. 2005). Although internal focusing strategies for regulating personally relevant NA likely involve medial prefrontal regions, we still thought it important to assure self-relevant mood induction.

Negative self-reference (SR) is central to NA (Davis and Unruh 1981; Rector et al. 1998) and to Beck's (1967) cognitive model of depression. Neuroimaging findings relate Negative SR to reciprocal limbic-cortical changes (Fossati et al. 2003; Gillihan and Farah 2005). No studies to-date have used video-based personal events to induce NA in an emotion regulation study.

\section{Aims of the study}

Our first purpose was to use functional magnetic resonance imaging (fMRI) to identify changes in regional cerebral activity associated with NA induced by viewing a selfreferent video of personal competitive failure. The next was to investigate the neural correlates of a brief cognitive intervention (CI) designed to cognitively reframe the event and resulting emotional response. Although this study intentionally did not explore the efficacy of a cognitive treatment due to sample size constraints, we nonetheless showed videos of a failed swim before and immediately after a single, 20-min CI. The central hypotheses were that negative SR would be associated with altered regional cerebral blood flow (rCBF) in the premotor, sensorimotor cortices as well as in the ventromedial and dorsomedial prefrontal cortices. Secondarily, we intended to use the CI to elucidate the importance of addressing cognitive perspective prior to video self-reference.

\section{Materials and methods}

\section{Subjects}

Fourteen elite athletes (ten males, ranging in age from 18 to 31, M $23.8 \pm 3.5$ years) took part in the study on the basis of having made major competitive errors that had resulted in career-threatening failure. Eleven participants failed the Olympic trials, missing qualification for the Olympic Games, and three athletes failed at the Olympic Games. One subject was eliminated because of technical problems during fMRI 
acquisition. For each athlete the fMRI session occurred at roughly 8 months following failure at Olympic trials.

None of these athletes had a history of having used medication for a mood disorder. The Beck Depression Inventory mean at the time of fMRI was $9.7 \pm 7.2$. As national team athletes, each had undergone regular testing for drug abuse under World Anti-Doping Protocols and none had ever tested positive for using banned substances. As well, no athlete had a history of a substance abuse disorder and none had been intoxicated with alcohol within at least the 2 weeks prior to fMRI. Written, informed consent was obtained from all participants according to ethics guidelines of the University of British Columbia.

\section{Tasks}

Each fMRI session consisted of a continuous, fixed order, 16-min acquisition in which neutral video viewing (4-min) always preceded failure video viewing (2 blocks of 4-min each). One-minute rest blocks preceded both video viewing conditions, and a 2-min rest block ended each session. Subjects rated on line their subjective level of NA throughout the fMRI sessions using a visual analogue scale $(0-7)$, present on the screen at all times, according to the instruction to "Use the response pad to indicate your current level of sadness." Using a fiber optic response device, they pressed one button to increase their rating of subjective distress (left index finger), and the second to decrease it (right index finger).

The neutral video condition displayed other athletes racing and was shown to enable a within-subject contrast against which to understand the failure video activations. Instructions were to watch the video and continuously monitor mood. The failure video condition showed a recent personal, failed performance. No athlete had viewed the 4-min video prior to fMRI.

Instructions varied with the fMRI session. At pre-CI (fail video1), both orally and on the viewing screen during fMRI the athletes were asked to re-experience the way they felt immediately during and after the failed performance. After the first fMRI session, athletes went to a nearby room for the three-part CI to: (1) Express feelings generated from watching the failed race; (2) Express self-referent cognitions related to the actual motor performance, which tended to be both attitudinal and behavioral (e.g., "I am slow", "I need to work on my stroke length"); (3) Consider and imagine performance changes for the next race. It should be remembered that the CI was not cognitive therapy, however, and that the CI was used as a between-session focusing exercise akin to a visual motor behavioral rehearsal (cf. Suinn 1985). At post-CI (fail video 2) subjects again rated affect by re-experiencing the way they felt during and after the performance according to the same instruction as that which had preceded video 1 .
Image acquisition

Echo-planar images were collected on a Philips Gyroscan Intera 3.0-T scanner, equipped with a SENSE coil. Conventional spin-echo $\mathrm{T}_{1}$-weighted sagittal localizers were used to view head position and to graphically prescribe the functional image volumes. Functional image volumes sensitive to the blood oxygen-level dependent (BOLD) contrast signal were collected with a gradient echo sequence (TR/TE $2000 / 30 \mathrm{~ms}, 90^{\circ}$ flip angle, field of view $210 \times 143 \times 240 \mathrm{~mm}$ (anteroposterior, feet-head, right-left), $3.00 \mathrm{~mm}$ slice thickness, slice gap $1 \mathrm{~mm}, 36$ axial slices.

\section{Image processing}

Statistical Parametric Mapping software (Wellcome Institute of Cognitive Neurology, http://www.fil.ion.ucl.ac.uk/ $\mathrm{spm} /$ ) was used for image reorientation, realignment, normalization into Montreal Neurological Institute space, and smoothing with a Gaussian kernel $(8 \mathrm{~mm}$ full width at half maximum) to compensate for inter-subject anatomical differences and optimize the signal to noise ratio. The BOLD response was modeled using finite impulse response (FIR) functions estimated for each of eight continuous 30-s epochs within each 4-min block. These epoch blocks permitted the evaluation of the a priori hypothesis that the BOLD response would correspond to mood ratings which had also been aggregated to 30 -s epochs.

Given the limited number of elite athletes, no group contrast to compare treated and untreated subjects was possible. While the main focus was on the comparison between viewing fail video 1 and fail video2 (isolating the regional effects linked to the variation of negative mood in response to the failed swim), the neutral video condition was added as control for test-retest repetition effects.

Voxel-by-voxel analysis The results of the FIR estimates were employed to test the contrast of fail video1 vs. fail video2. The significance of the contrast was tested using a random effects model in which a single image of the contrast of interest is brought forward for each subject. The random effects analysis height threshold was first set at a more conservative, wholebrain correction level $(t=3.56, p<0.005)$. A second, more liberal significance threshold was used to test regional effects expected on an a priori basis for areas previously implicated in negative mood and SR $(t=1.78, p<0.05$, uncorrected for multiple comparisons).

ROI post-hoc analyses Binary masks were computed to show significantly increased or decreased activity during fail video1 and fail video2. First, an individual subject's mean beta value was extracted for each 4-min condition (neutral video pre-CI, fail video1, neutral video post-CI, fail 
video2). Mean beta values for each region of interest (ROI) were entered in two-way repeated measures analyses of variance (ANOVAs) with session (pre- and post-CI) and condition (neutral video vs. fail video). For each of the above defined regional clusters, we assessed the relationship between the brain activity pre-CI and post-CI and the subjective intensity of the induced mood in these periods. Beta values were determined for each subject at each 30-s epoch, and this value was correlated with the durationweighted mean subjective mood rating for that epoch (allowed by the FIR analysis) using simple correlations.

\section{Results}

Mood measures

Mean subjective ratings were averaged across each condition, and a two-way repeated measures ANOVA was conducted. There were main effects of session (pre-CI vs. post-CI), $F(1,24)=29.4, p<0.0001$ ), and video (neutral vs. fail), $F(1,24)=33.3, p<0.0001$. These were qualified by the significant interaction of session $\times$ video, $F(1,24)=18.4$, $p=0.002$. Mean sadness ratings were $3.8 \pm 0.04$ for the fail video1 and $1.1 \pm 0.2$ for the neutral video pre-CI; $1.7 \pm 0.3$ for the fail video2, and $1.0 \pm 0.2$ for the neutral video postCI. Post-hoc comparisons revealed that athletes' ratings were higher for fail videol than for the neutral pre-CI video, $F(1,12)=33.7, p<0.0001$ and for fail video 2 than neutral post-CI video, $F(1,12)=7.6, p=0.02$. However, critically, the fail videol mood ratings were higher than the fail video2 ratings, $F(1,12)=36.1, p<0.0001$, confirming that reviewing the fail swims provoked substantially more NA prior to CI than after applying the CI instructions. See Fig. 1 for a plot of the mean mood ratings as a function of condition and fMRI scan time. Importantly, as a control for stimulus habituation each ROI contrast compared the response to viewing personal failure with the response to viewing a neutral stimulus. ROI contrasts comparing neutral stimulus BOLD activity pre-CI to post-CI showed no significant changes as outlined below. The literature consistently shows reliability estimates of using visual analog scale (VAS) to estimate NA in the range of 0.9 and reports strong validity when VAS ratings are correlated to standard profiles of mood (Lingjaerde and Foreland 1998; Steiner and Streiner 2005).

Voxel-by-voxel analysis

Fail video2 $>$ fail video1 Significantly greater BOLD activity post-CI relative to pre-CI (height threshold $t=3.56$, $p<0.005$, corrected, cluster threshold size $=300$ ) included

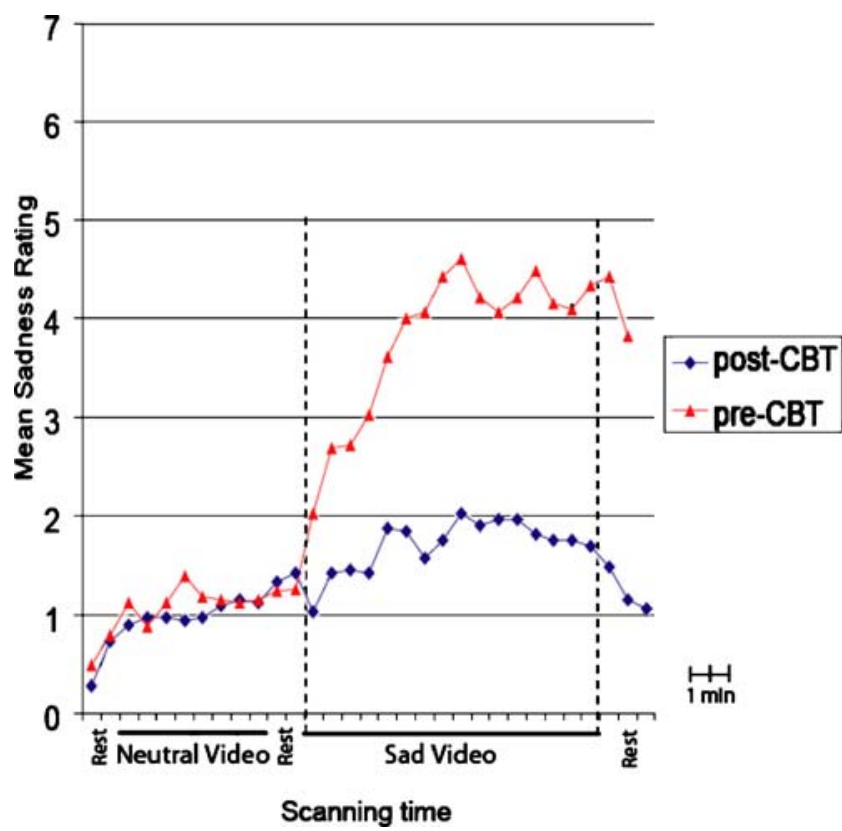

Fig. 1 Mean sadness ratings as a function of time in the MRI scanner in the pre and post-CI sessions. Note increase in the ratings during the fail videos

two clusters in right premotor cortex BA6 and left sensorimotor cortex, BA4/1 (see Table 1, top).

Fail Video1 $>$ Fail Video2 Significantly greater BOLD activity before than after CI (height threshold $t=1.78, p<$ 0.05 , uncorrected, cluster threshold size $=400$ ) included three clusters in right parahippocampal gyrus (Brodmann areas (BA 28/36), ventral medial PFC and adjacent rostral anterior cingulate cortex (BA10/32) and dorsomedial and dorsolateral PFC (BAs 10, 32 and 45; see Table 1, bottom, and Fig. 2).

\section{ROI analysis}

Right premotor cortex BA6 Activity during the fail video2 was greater than during fail videol $(p<0.001)$. Pre-CI, activity in this region was significantly less during fail video1 than neutral video $(p<0.003)$. In contrast, post-CI, activity was not dissimilar for fail video 2 and neutral video, and neither differed from the pre-CI neutral video (see Figs. 2 and 3 ).

Left sensorimotor cortex $B A 4$ and 1 Similarly, fail video2 yielded greater activity than fail videol $(p<0.001)$, and this was mostly explained by less activity during the fail video 1 relative to the neutral video $(p<0.07)$, while post-CI activity was similar for fail video 2 and neutral video, and at the same levels as the neutral viewing pre-CI. 
Table 1 Clusters of significant BOLD signal change between fail video1 and fail video2

\begin{tabular}{|c|c|c|c|c|}
\hline Region & Brodmann area & Cluster size & Peak $t$ value & Coordinates \\
\hline \multicolumn{5}{|c|}{ Fail video $2>$ fail video $1^{\mathrm{a}}$} \\
\hline R Pm & 6 & 369 & $\begin{array}{l}5.54 \\
4.82 \\
5.14\end{array}$ & $\begin{array}{l}26,1,42 \\
20,-1,47 \\
15,6,46\end{array}$ \\
\hline $\mathrm{L} \mathrm{M} 1 / \mathrm{S} 1$ & 4 and 1 & 308 & $\begin{array}{l}5.32 \\
4.31 \\
3.98\end{array}$ & $\begin{array}{l}-29,-17,43 \\
-24,-15,55 \\
-34,-21,53\end{array}$ \\
\hline \multicolumn{5}{|c|}{ Fail video $1>$ fail video $2^{\mathrm{b}}$} \\
\hline R GH & 28 and 36 & 448 & $\begin{array}{l}3.69 \\
3.33 \\
3.30\end{array}$ & $\begin{array}{l}17,-19,-21 \\
27,-23,-21 \\
22,-29,-28\end{array}$ \\
\hline vMF & 10 & 631 & 3.42 & $-8,53,3$ \\
\hline $\mathrm{GC}$ & 32 & & $\begin{array}{c}2.79 \\
2.76\end{array}$ & $\begin{array}{c}17,55,6 \\
-1,49,8\end{array}$ \\
\hline $\begin{array}{l}\mathrm{R} \text { dorsomedial PFC } \\
\mathrm{R} \text { dorsolateral PFC }\end{array}$ & $\begin{array}{l}32 \text { and } 10 \\
45\end{array}$ & 885 & $\begin{array}{l}3.35 \\
3.23 \\
2.94\end{array}$ & $\begin{array}{l}17,28,29 \\
48,26,10 \\
24,51,22\end{array}$ \\
\hline
\end{tabular}

$R$ Right, $L$ left, $P m$ premotor, $M 1$ primary motor, $S 1$ primary sensory, $G H$ parahippocampal gyrus, $v M F$ ventromedial PFC, $G C$ cingulate gyrus, $P F C$ prefrontal cortex.

${ }^{\text {a }}$ For fail video $2>$ fail video1, height threshold is $t=3.56, p<0.005$, corrected, cluster threshold $=300$

${ }^{\mathrm{b}}$ For Fail Video1 $>$ Fail Video2, height threshold is $t=1.78, p<0.05$, uncorrected, cluster threshold $=400$

Fig. 2 Top: significant clusters where BOLD signal is greater for fail video 2 than fail video 1 $(t>3.56, p<0.005$, uncorrected). $\mathrm{R}$ Pm6 $=$ right premotor cortex BA6; L M1/S1 = left primary motor/sensory cortex BA4\&1. Bottom: significant clusters where BOLD signal is greater in the fail video 1 than the fail video2 $(t>1.78, p<0.05$, uncorrected). On the left, activation in ventromedial PFC BA10 (vMF10) and cingulate gyrus BA32 (GC32). On the right, activation in right parahippocampal gyrus BA36 (GH36) and dorsomedial PFC BA10 (dMF10)
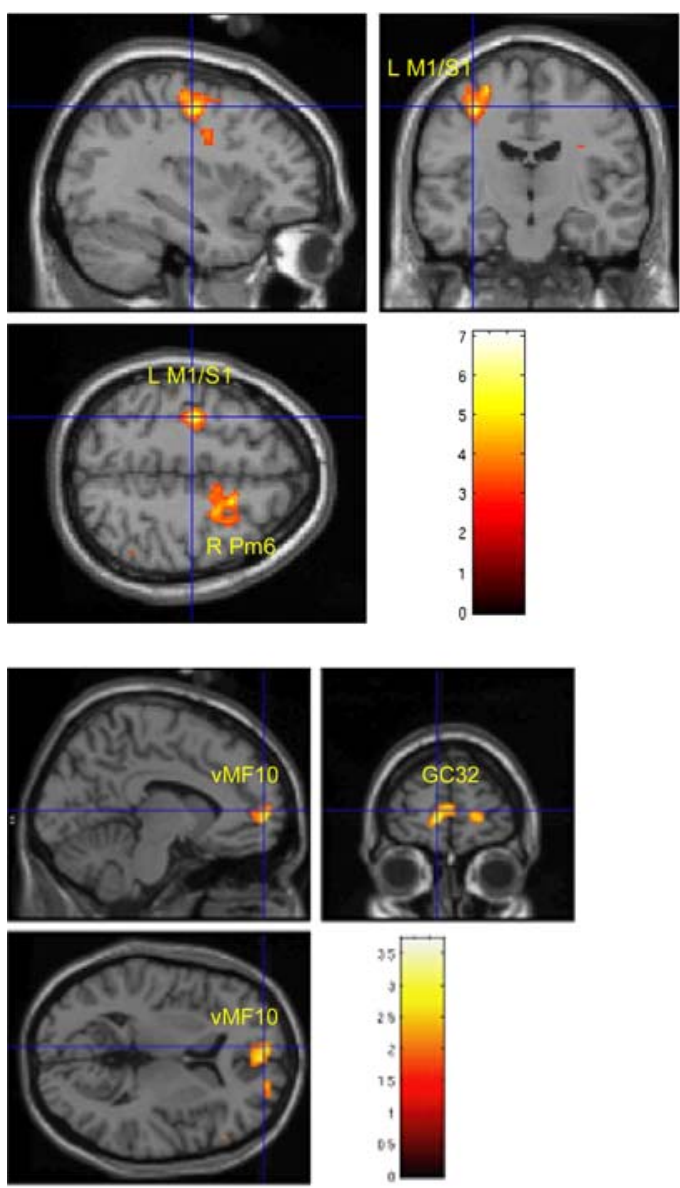
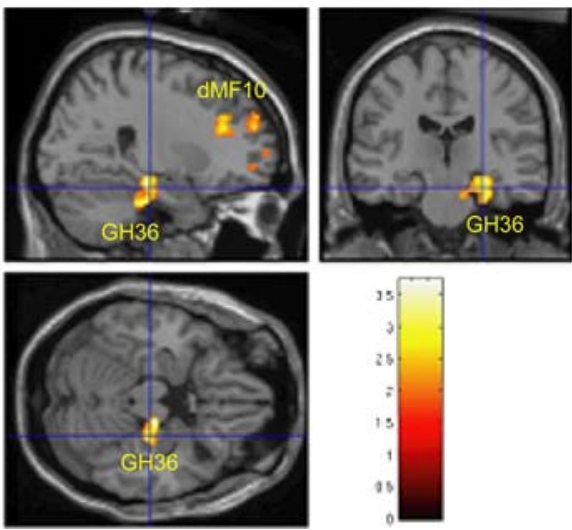
Fig. 3 Mean percent signal change in $\mathrm{R}$ premotor BA6 (Pm6, left) and in L M1/ S1 (right) while watching fail and neutral videos pre and post-CI. Note selectively decreased activity during fail video pre-CI

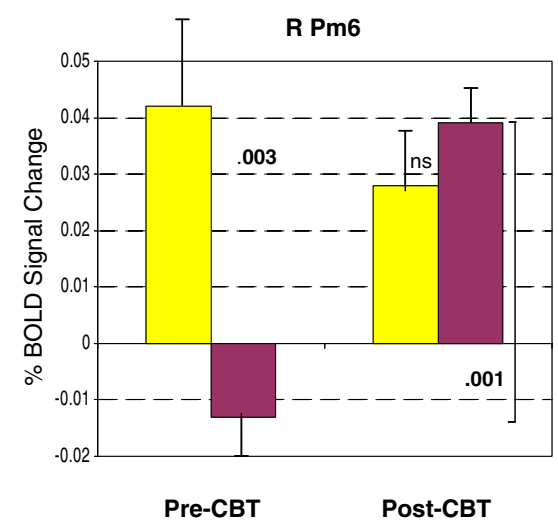

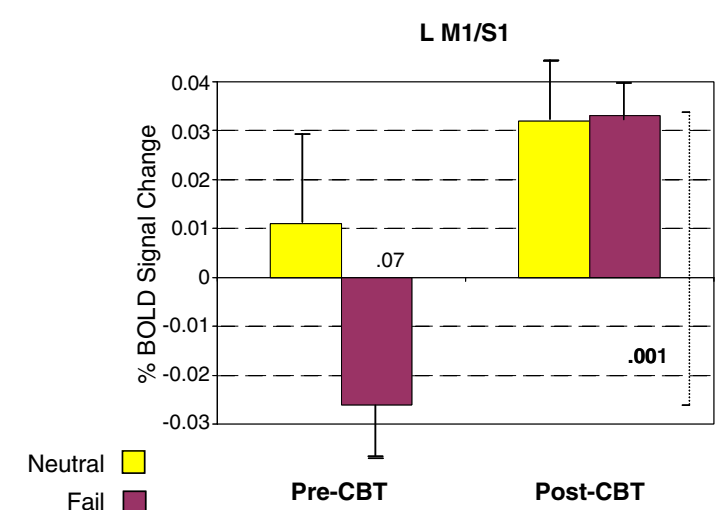

Ventromedial PFC BA10 and 32 Greater activity was present for fail video1 than fail video $2(p<0.016)$. This effect was contributed by a relative increase over neutral video pre-CI, and a reverse-sign, relative decrease in activity post-CI. However, neither of these changes over the neutral conditions reached significance (see Fig. 4).

Right dorsomedial (BA10) and right dorsolateral PFC (BA45) Similarly, greater activity was evident pre-CI than post-CI $(p<0.008)$, and this was explained by a relative increase over neutral viewing pre-CI (not significant), and a reverse-sign, relative decrease in activity post-CI $(p=0.10)$.

Right parahippocampal gyrus Fail video viewing elicited significantly more BOLD activity pre-CI than post-CI ( $p<$ $0.001)$. This difference was explained by a different pattern than for the other ROIs. BOLD activity in right parahippocampal gyrus $(\mathrm{GH})$ was greater when the Fail videos were shown relative to the neutral videos both pre-CI ( $p<$ $0.001)$ and post-CI $(p<0.0005$; see Fig. 3).

\section{Correlations}

BOLD signal in the right premotor cortex BA6 was negatively correlated to sadness intensity $(p<0.01)$, with greater reported NA associated to lower premotor activity. Non-significant positive correlations were found with BOLD activity in the right parahippocampal region $(p<0.10)$ and ventromedial PFC $(p<0.25)$.

Simple correlations of BOLD activity between ventromedial PFC and the other frontal regions (R Pm6, L M1/S1 and $\mathrm{R}$ PFC) were positive with the exception of failure viewing (pre-CI) which was negative for R Pm6 and, particularly, L M1/S1 (see Fig. 5).

\section{Discussion}

During fMRI, athletes first engaged in negative SR by watching their failed races. This resulted in NA, as predicted by Beck's (1967) cognitive model of depression. After a brief $\mathrm{CI}$, they then viewed the failed performance again, and this gave rise to considerably less NA. Selfreferential reviewing of failure activated ventromedial $\mathrm{PFC}$, including rostral ACC, dorsomedial and dorsolateral PFC, and the right parahippocampus, while a marked reduction of activity was observed in motor-premotor areas.

Limbic effects

The changes in ventromedial prefrontal cortex and parahippocampus in the present study replicate previous findings in mood provocation and SR studies, consistent
Fig. 4 Mean percent signal change in vMF10/GC32 (left) and in GH36/28 (right) while watching fail and neutral videos pre and post-CI
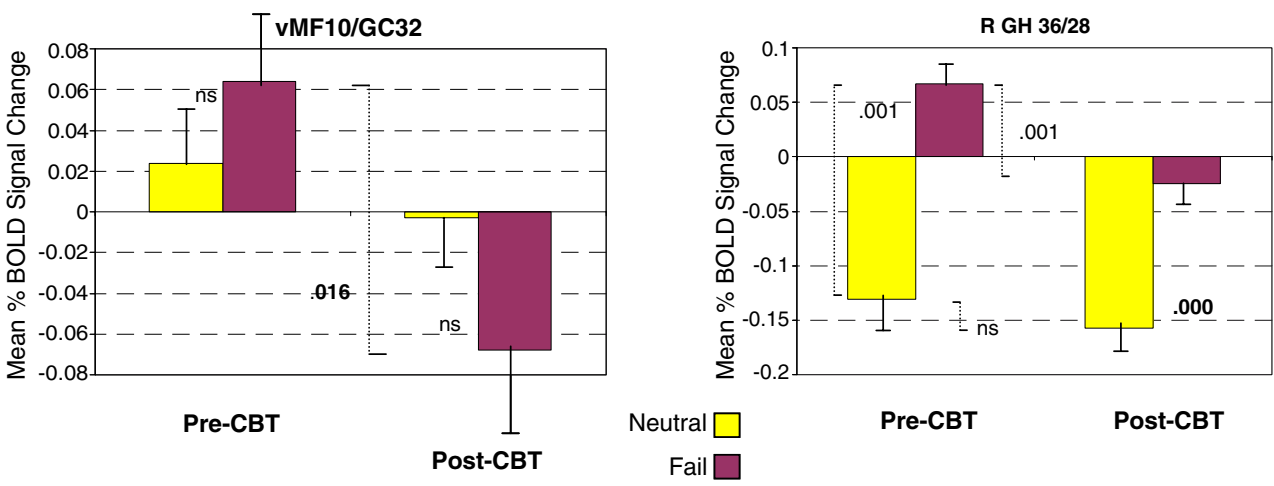




Fig. 5 Correlation of BOLD signal change between MF10/GC32 and RPm6 (left), LM1/S1 (center) and R PFC (right) for each session (pre$\mathrm{CI}$, post-CI) and Video (neutral, fail). Note negative sign correlations in the fail 1 video only for RPm6 and LM1/S1

with role of these regions in the expression and regulation of negative emotion (George et al. 1995; Gotlib et al. 2005; Lane et al. 1997; Mayberg et al. 1999; Phan et al. 2005). A number of previous studies have employed sad stimuli and asked subjects to make self-referential emotion-judgments. The majority of such studies have not employed personally salient or autobiographical stimuli. For example, Keedwell et al. (2005) provoked SR to enhance the recall of sad events in clinical depressives by first showing a photo of sad third-persons. This resulted in activation of the prefrontal cortex (BA9) and posterior cingulate cortex (BA31). Gusnard and colleagues (2001) asked healthy subjects to make self-referential emotion-judgments while reviewing sad and happy photos; they reported increased activity in medial prefrontal cortex (BA 8 and 10). An exception are studies employing autobiographical sad memories to provoke SR and elicit sadness (Bechara et al. 2000; Liotti et al. 2000, 2002). Consistent with such studies, our subjects reviewed their own NA-evoking failures before planning and imagining corrections. Our results suggest that when negative schemas are invoked by focusing on personal failure, medial prefrontal cortex is activated, while motor-premotor regions are concomitantly suppressed, as testified by the significant negative correlations in Fig. 5 (pre-CI). Conversely, focusing on behavioral possibility rather than on failure (post-CI), resulted in relatively less activation of the $\mathrm{mPFC}$, and allowed restored activation of premotor-motor regions. This is to our knowledge the first study reporting reciprocal sign effects in motor-premotor cortex during viewing of own-action associated to SR and NA.

Activation in the right parahippocampal gyrus in the present study is consistent with a possible role in generating defensive responses to aversive stimuli, suggested by fMRI studies employing faces, music, and films (Lane et al. 1997), of aversive nature, as well as lesion correlation data showing that right $\mathrm{GH}$ lesions impair the ability to recognize dissonant music as unpleasant (Gosselin et al. 2006).

Motor-premotor effects

Previous studies reported similar reciprocal sign changes (less activity) associated to negative mood, but such rCBF reductions were in right dorsolateral PFC (BA9/46) rather than in motor-premotor cortex (Mayberg et al. 1999). A fMRI study reported similarly reduced blood flow in left dorsolateral PFC relative to healthy subjects in remitted depressives listening to criticism from their own mothers (Hooley et al. 2005). Dorsolateral PFC subserves attention and working memory functions, and its hypoactivation may relate to the cognitive deficits observed in depressed individuals (Liotti and Mayberg 2001). To our knowledge, the present study is the first following Vogeley et al. (2001) to have demonstrated similar reciprocal changes in motor-premotor regions during mood induction and negative SR. Such reductions where inversely correlated with the activation of ventromedial $\mathrm{PFC}$ and the subjective ratings of NA, emphasizing the link between NA and premotor function. Reduced activity in premotor-motor areas may represent a substrate for the motor retardation/slowness observed in depressed patients (e.g., Sabbe et al. 1999). In the context of the present study, motor-premotor areas appear to be logically responsible for motor programming and action planning, necessary pre-requisites of competitive readiness. It is important to note that failed action observation and perhaps motor imagery may be necessary to bring about such reciprocal sign effects of SR and NA, which may explain why they were not reported previously.

Earlier self-reference studies have not reported effects in the motor system. Voluntary suppression of negative affect has been found to reduce posterior cingulate activity, but no changes in motor-premotor cortex were reported (Phan et al. 2005). Similarly, Beauregard et al. (2001) employed suppression methods but did not focus on behavior and did not show motor cortex findings. Ochsner et al. (2002, 2004), required a review of a sad third-person and demonstrated $\mathrm{mPFC}$ activity in the absence of significant motor-premotor activations. Comparing the present results to those of Ochsner, it appears that first-person as opposed to third-person self-reference leading to NA is essential to bring about the premotor effects. In addition, action processing is key to the design because, although information processing of personal adjectives requires explicit SR, adjective processing itself may have little to do with motor activity.

In summary, it is likely that only action-based, personal self-referential material brings about effects on the motor system. Consistent with this suggestion, Amodio and Frith 
(2006) propose that self-monitoring that requires action monitoring uniquely invokes processing in an area that is contiguous to our BA6.

Outside the area of self-reference, activation of motorpremotor areas has been reported during action preparation and imagery (Marshall et al. 1997; Rodriguez et al. 2004; Wraga et al. 2005), and during action observation. For example, studies in dance indicate that watching another dancer while referencing the observed performance against one's own repertoire can activate parietal, premotor, and motor cortices (Cross et al. 2006). However, human movement studies that are not self-referent do not appear to show the PFC-premotor correlated activations (Castelli et al. 2000; Nyberg et al. 2006). Gusnard et al. (2001) reported a tandem increase in medial PFC and premotor BA6 activity during observation of action-oriented emotional pictures (skydivers, burn victims, guns pointed at the subject) likely contributing to premotor activation. Interestingly, no significant parahippocampal activations were found in that study, perhaps suggesting that stimuli overall did not produce a significant aversive response. By looking at the medial PFC-premotor correlations in the present study, it is clear that concordant, positive sign changes in the two regions are observed when self-referent emotional material is not accompanied by NA - as in Gusnard et al. (2001) and Amodio and Frith's (2006) study-while reciprocal sign changes are only observed when selfreferent material produces significant negative affect (pre$\mathrm{CI}$ ). We propose here that medial PFC-premotor functional connectivity may prompt motor responses to self-relevant stimuli, perhaps part of an adaptive response of survival value - to prepare the organism to fight or flight. However, in the presence of a build-up of significant sadness or NA, such functional connectivity would revert, with reciprocal sign changes in $\mathrm{mPFC}$ and premotor areas. We speculate that such behavioral pattern in response to perceived failure may correspond to what is observed during learned helplessness when the animal stops trying to escape and simply lies still (Panksepp 1998).

Another area of research that may be relevant to our findings is the one associating medial PFC to error processing, including error monitoring and detection, error compensation, and response to negative feedback (e.g., Nieuwenhuis et al. 2005; Yeung et al. 2004). Late, conscious error compensation includes post-error slowing in an attempt to improve future performance. However, these studies report event-related activity elicited by button press errors during choice reaction time tasks, rather than activity evoked by action observation of themselves making errors. While it is possible that the same system is engaged during self-error observation, there is no available evidence to-date to support such a claim.

\section{Effects of CI}

Unlike previous research of cognitive appraisal or voluntary suppression of emotion, in the present study we did not attempt deliberate affect inhibition or cognitive distraction (Ochsner et al. 2002, 2004). Subjects were instructed to notice and allow affect while simultaneously considering and imagining how to modify their technique, power generation and strategy in order to create a more successful future motor performance, if they would be able to redo the race. Therefore our SR provocation was distinctive because it involved referencing both performance and affect. Our athlete subjects had arguably over-learned their motor skills and were among the best in the world in executing these skills at competition. Nevertheless, because of the extreme level of competition in elite swimming, they happen to fail. No personality testing was conducted to shed light on whether some subjects were more affectively activated by self-reference. This possibility will be explored in future work with larger samples. Each athlete had prior experience with managing performance-generated NA, but some may still have perceived that their personal failure was of greater significance than that of others. This potentiality underscores the importance of our having contrasted the athletes individual SR responses to their own neutral stimulus responses.

\section{Conclusion}

In summation, we showed expected increases in ventral mPFC and parahippocampal activity as mood worsened with SR mood induction; these changes were opposite to reciprocal sign premotor-motor changes. A brief CI strategy which allowed for the consideration of new, corrective motor behavior showed potential for reversing both the neural correlates of NA and debilitating effects of NA on sport performance. Future work will incorporate experimental controls by contrasting neural activity in two groups - failed and not-failed non-elite athletesundergoing the same SR task. The use of non-elite athletes will permit larger samples and obviate a withinsubject design.

This suggests that suppression of activity in motor regions during SR may be an important marker of failed sport performance. We hypothesize that athletes at risk for repeated failures will show the greatest decrements in premotor activity, while more resilient athletes may exhibit activation of premotor cortex as a result of self-action observation. The at risk athletes should be the ones to derive the greatest benefits from a CI based on reviewing of the failing performance. 
Acknowledgement The principal funding for this research and for the work of the first author, Dr. H. Davis came from private donations to Swimming Canada. The authors extend special thanks to the participating Canadian athletes and their coaches for unqualified support of this work. Dr. Burkhard Mädler of the University of British Columbia MRI Research Centre is thanked for facilitating the completion of this work. Thanks are also extended to Drs. Tricia Orzeck and Patrick Baillie for assistance though out the project. Supplementary to this was funding to the second author, Dr. Mario Liotti, from the National Alliance for Research on Schizophrenia and Depression and the Canada Foundation for Innovation. Correspondence concerning this article should be addressed to Dr. Hap Davis, Swimming Canada, Suite 354, 401-9th Avenue S.W., Calgary, AB, Canada. E-mail: hapdavis@gmail.com

Conflict of interest statement There were no biomedical financial conflicts of interests and there were no conflicts of interest, directly or indirectly, for any of the researchers to declare.

\section{References}

Amodio, D. M., \& Frith, C. D. (2006). Meeting of minds: The medial frontal cortex and social cognition. Nature reviews. Neuroscience, 7(4), 268-277.

Baillie, P. H., \& Danish, S. J. (1992). Understanding the career transition of athletes. Sport Psychologist, 6(1), 77-98.

Beauregard, M., Levesque, J., \& Bourgouin, P. (2001). Neural correlates of conscious self-regulation of emotion. The Journal of Neuroscience: The Official Journal of the Society for Neuroscience, 21(18), $\mathrm{RC} 165$.

Bechara, A., Damasio, H., \& Damasio, A. R. (2000). Emotion, decision making and the orbitofrontal cortex. Cerebral Cortex, 10(3), 295-307.

Beck, A. T. (1967). Depression. Harper and Row: New York.

Beedie, C. J., Terry, P. C., \& Lane, A. M. (2000). The profile of mood states and athletic performance: Two meta-analyses. Journal of Applied Sport Psychology, 12(1), 49-68.

Carrm, C., Carter, J., Beck, N., Chamberlain, R., \& Zaichkowsky, L. D. (2005). Addressing the psychological health issues of the collegiate student-athlete. In M. E. Ewing (President). Association for the Advancement of Applied Sport Psychology: Conference Proceedings, Vancouver, British Columbia, Canada, 35, pp. 793-797.

Castelli, F., Happe, F., Frith, U., \& Frith, C. (2000). Movement and mind: A functional imaging study of perception and interpretation of complex intentional movement patterns. NeuroImage, 12 (3), 314-325.

Cross, E. S., Hamilton, A. F., \& Grafton, S. T. (2006). Building a motor simulation de novo: Observation of dance by dancers. NeuroImage, 31(3), 1257-1267.

Davis, H., \& Unruh, W. R. (1981). The development of the selfschema in adult depression. Journal of Abnormal Psychology, 90 (2), 125-133.

Eugene, F., Levesque, J., Mensour, B., Leroux, J. M., Beaudoin, G., Bourgouin, P., et al. (2003). The impact of individual differences on the neural circuitry underlying sadness. NeuroImage, 19(2, Pt 1), 354-364.

Fazackerley, R., Lane, A. M., \& Mahoney, C. (2004). Mood and performance relationships in wakeboarding. Journal of Sport Behavior, 27(1), 18-30.

Fossati, P., Hevenor, S. J., Graham, S. J., Grady, C., Keightley, M. L., Craik, F., et al. (2003). In search of the emotional self: An fMRI study using positive and negative emotional words. American Journal of Psychiatry, 160(11), 1938-1945.

George, M. S., Ketter, T. A., Parekh, P. I., Horwitz, B., Herscovitch, P., \& Post, R. M. (1995). Brain activity during transient sadness and happiness in healthy women. The American Journal of Psychiatry, 152(3), 341-351.

Gillihan, S. J., \& Farah, M. J. (2005). Is self special? A critical review of evidence from experimental psychology and cognitive neuroscience. Psychological Bulletin, 131(1), 76-97.

Gosselin, N., Samson, S., Adolphs, R., Noulhiane, M., Roy, M., Hasboun, D., et al. (2006). Emotional responses to unpleasant music correlates with damage to the parahippocampal cortex. Brain: A Journal of Neurology, 129(Pt 10), 2585-2592.

Gotlib, I. H., Sivers, H., Gabrieli, J. D., Whitfield-Gabrieli, S., Goldin, P., Minor, K. L., et al. (2005). Subgenual anterior cingulate activation to valenced emotional stimuli in major depression. Neuroreport, 16 (16), 1731-1734.

Gusnard, D. A., Akbudak, E., Shulman, G. L., \& Raichle, M. E. (2001). Medial prefrontal cortex and self-referential mental activity: Relation to a default mode of brain function. Proceedings of the National Academy of Sciences of the United States of America, 98(7), 4259-4264.

Hooley, J. M., Gruber, S. A., Scott, L. A., Hiller, J. B., \& Yurgelun-Todd, D. A. (2005). Activation in dorsolateral prefrontal cortex in response to maternal criticism and praise in recovered depressed and healthy control participants. Biological Psychiatry, 57(7), 809-812.

Keedwell, P. A., Andrew, C., Williams, S. C., Brammer, M. J., \& Phillips, M. L. (2005). The neural correlates of anhedonia in major depressive disorder. Biological Psychiatry, 58(11), 843-853.

Lane, R. D., Reiman, E. M., Ahern, G. L., Schwartz, G. E., \& Davidson, R. J. (1997). Neuroanatomical correlates of happiness, sadness, and disgust. The American Journal of Psychiatry, 154 (7), 926-933.

Lane, A. M., Terry, P. C., Beedie, C. J., Curry, D. A., \& Clark, N. (2001). Mood and performance: Test of a conceptual model with a focus on depressed mood. Psychology of Sport and Exercise, 2(3), 157-172.

LeUnes, A., \& Burger, J. (2000). Profile of mood states research in sport and exercise psychology: Past, present and future. Journal of Applied Sport Psychology, 12(1), 5-15.

Lingjaerde, O., \& Foreland, A. R. (1998). Direct assessment of improvement in winter depression with a visual analog scale: High reliability and validity. Psychiatry Research, 81(3), 387-392.

Liotti, M., \& Mayberg, H. S. (2001). The role of functional neuroimaging in the neuropsychology of depression. Journal of Clinical and Experimental Neuropsychology: Official Journal of the International Neuropsychological Society, 23(1), 121-136.

Liotti, M., Mayberg, H. S., Brannan, S. K., McGinnis, S., Jerabek, P., \& Fox, P. T. (2000). Differential limbic-cortical correlates of sadness and anxiety in healthy subjects: Implications for affective disorders. Biological Psychiatry, 48(1), 30-42.

Liotti, M., Mayberg, H. S., McGinnis, S., Brannan, S. L., \& Jerabek, P. (2002). Unmasking disease-specific cerebral blood flow abnormalities: Mood challenge in patients with remitted unipolar depression. The American Journal of Psychiatry, 159(11), 1830-1840.

Marshall, J. C., Halligan, P. W., Fink, G. R., Wade, D. T., \& Frackowiak, R. S. (1997). The functional anatomy of a hysterical paralysis. Cognition, 64, B1-8.

Mayberg, H. S., Liotti, M., Brannan, S. K., McGinnis, S., Mahurin, R. K., Jerabek, P. A., et al. (1999). Reciprocal limbic-cortical function and negative mood: Converging PET findings in depression and normal sadness. The American Journal of Psychiatry, 156(5), 675-682.

Nieuwenhuis, S., Slagter, H. A., von Geusau, N. J., Heslenfeld, D. J., \& Holroyd, C. B. (2005). Knowing good from bad: Differential activation of human cortical areas by positive and negative 
outcomes. The European Journal of Neuroscience, 21(11), 31613168.

Northoff, G., Heinzel, A., de Greck, M., Bermpohl, F., Dobrowolny, H., \& Panksepp, J. (2006). Self-referential processing in our brain-a meta-analysis of imaging studies on the self. NeuroImage, 31(1), 440-457.

Nyberg, L., Eriksson, J., Larsson, A., \& Marklund, P. (2006). Learning by doing versus learning by thinking: An fMRI study of motor and mental training. Neuropsychologia, 44(5), 711-717.

Ochsner, K. N., Bunge, S. A., Gross, J. J., \& Gabrieli, J. D. (2002). Rethinking feelings: An FMRI study of the cognitive regulation of emotion. Journal of Cognitive Neuroscience, 14(8), 1215-1229.

Ochsner, K. N., Ray, R. D., Cooper, J. C., Robertson, E. R., Chopra, S., Gabrieli, J. D., et al. (2004). For better or for worse: Neural systems supporting the cognitive down- and up-regulation of negative emotion. NeuroImage, 23(2), 483-499.

Ochsner, K. N., Beer, J. S., Robertson, E. R., Cooper, J. C., Gabrieli, J. D., Kihsltrom, J. F., et al. (2005). The neural correlates of direct and reflected self-knowledge. NeuroImage, 28(4), 797-814.

Panksepp, J. (1998). Affective neuroscience: the foundations of human and animal emotions. New York: Oxford University Press.

Phan, K. L., Magalhaes, A., Ziemlewicz, T. J., Fitzgerald, D. A., Green, C., \& Smith, W. (2005). Neural correlates of telling lies: A functional magnetic resonance imaging study at 4 tesla. Academic Radiology, 12(2), 164-172.

Rector, N. A., Segal, Z. V., \& Gemar, M. (1998). Schema research in depression: A Canadian perspective. Canadian Journal of Behavioural Science, 30(4), 213-224.
Rodriguez, M., Muniz, R., Gonzalez, B., \& Sabate, M. (2004). Hand movement distribution in the motor cortex: The influence of a concurrent task and motor imagery. NeuroImage, 22(4), 1480-1491.

Sabbe, B., Hulstijn, W., van Hoof, J., Tuynman-Qua, H. G., \& Zitman, F. (1999). Retardation in depression: Assessment by means of simple motor tasks. Journal of Affective Disorders, 55(1), 39-44.

Sanders, C. E., Field, T. M., Diego, M., \& Kaplan, M. (2000). Moderate involvement in sports is related to lower depression levels among adolescents. Adolescence, 35(140), 793-797.

Steiner, M., \& Streiner, D. L. (2005). Validation of a revised visual analog scale for premenstrual mood symptoms: Results from prospective and retrospective trials. Canadian Journal of Psychiatry, 50(6), 327-332.

Suinn, R. M. (1985). Imagery rehearsal applications to performance enhancement. The Behavior Therapist, 8, 155-159.

Totterdell, P., \& Leach, D. (2001). Negative mood regulation expectancies and sports performance: An investigation involving professional cricketers. Psychology of Sport and Exercise, 2(4), 249-265.

Vogeley, K., Bussfeld, P., Newen, A., Herrmann, S., Happe, F., Falkai, P., et al. (2001). Mind reading: Neural mechanisms of theory of mind and self-perspective. NeuroImage, 14(1, Pt 1), 170-181.

Wraga, M., Shephard, J. M., Church, J. A., Inati, S., \& Kosslyn, S. M. (2005). Imagined rotations of self versus objects: An fMRI study. Neuropsychologia, 43(9), 1351-1361.

Yeung, N., Cohen, J. D., \& Botvinick, M. M. (2004). The neural basis of error detection: Conflict monitoring and the error-related negativity. Psychological Review, 111(4), 931-959. 